\title{
Detecting Outbreaks in Time-Series Data with RecentMax
}

\author{
Dave Carter* and Joel D. Martin \\ National Research Council Canada, Ottawa, ON, Canada
}

\section{Objective}

To develop an algorithm for detecting outbreaks of typical transmissible diseases in time series data that offers better sensitivity and specificity than the CDC EARS $\mathrm{C} 1 / \mathrm{C} 2 / \mathrm{C} 3$ algorithms while offering much better noise handling performance.

\section{Introduction}

We implemented the CDC EARS algorithms in our DADAR (Data Analysis, Detection, and Response) situational awareness platform. We encountered some skepticism among some of our partners about the efficacy of these algorithms for more than the simplest tracking of seasonal flu.

We analyzed several flu outbreaks observed in our data, including the H1N1 outbreaks in 2009, and noted that, using the C1 algorithm, even with our adjustable alerting thresholds, there was an uncomfortable number of false alarms in the noisy steady-state data, when the number of reported cases of flu-like symptoms was less than five per day.

We developed an algorithm, RecentMax, that could offer better performance in analyzing our flu data.

\section{Methods}

We developed the RecentMax algorithm based on a simple observation: that a good alert for an outbreak tends to be on days (or, more generally, in time slices) where the number of cases today is noticeably larger than on any recent day. For example, intuitively, if the number of cases of flu today is more than twice the greatest number of cases observed on any single day in the last three weeks, it seems reasonable to generate an alert.

RecentMax takes two parameters: a window of recent history (e.g., ten days), and a theshold factor (e.g. 1.5x), and generates alerts when the data observed for the current time slice is greater than the maximum observed in the history by the theshold factor, subject to the confidence interval (a tuneable probability of false positive threshold, typically set to $95 \%$ or higher, assuming a gaussian distribution around the threshold value).

\section{Results}

Our RecentMax algorithm does a commendable job alerting at the onset of the observed outbreaks.

Furthermore, RecentMax does a better job handing the noisy steady-state behaviour outside of outbreaks, offering intuitive alerts when the number of reported cases starts increasing meaningfully. The H1N1 outbreak of the fall of 2009 is illustrated using real-world data in the accompanying graphs; red bars highlight days on which CDC EARS C1 would generate alerts (at 95\% and 99\% confidence intervals) and days on which RecentMax would generate alerts given a 10-day history window and a threshold factor of $1.5 x$.

An interesting side-benefit of RecentMax is that, as an outbreak spreads and the number of cases increases somewhat linearly, the algorithm is less likely to generate alerts; that is to say, once an outbreak has been observed and many alerts have been generated (and presumably validated), the algorithm tends to stop alerting (unless the number of cases is increasing exponentially). Thus, once public health personnel have been notified of an outbreak in its early phases, the algorithm tends to not keep alerting, thus not telling recipients what they already know.

RecentMax avoids problems occasionally observed in algorithms built around a decay series. RecentMax examines the recent history without providing extra weight to more recent data, and is thus unaffected by noise in the immediately preceding time slice that can confuse some algorithms unduly (e.g., when data increases steadily for six consecutive days and then drops to zero on the seventh as the facility is closed for a holiday).

\section{Conclusions}

RecentMax offers a compelling alternative to the CDC EARS algorithms for detecting outbreaks of transmissible disease in timeseries data.

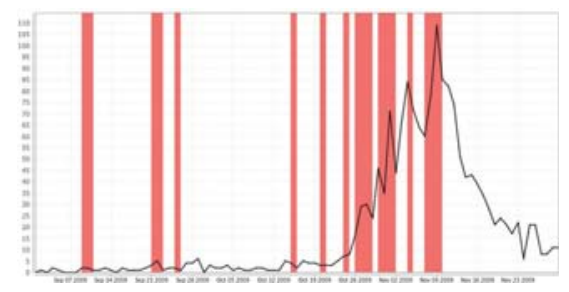

Flu outbreak with alerts generated by CDC EARS C1 at $95 \%$ confidence threshold

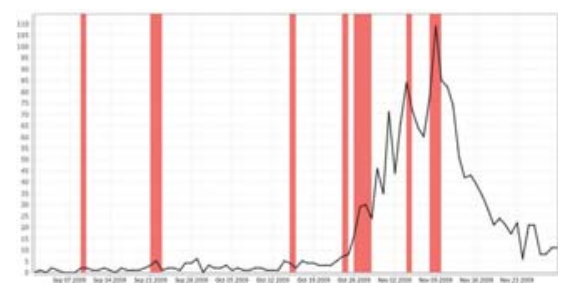

$2009 \mathrm{H} 1 \mathrm{H} 1$ outbreak with alerts generated by CDC EARS C1 at $99 \%$ confidence threshold

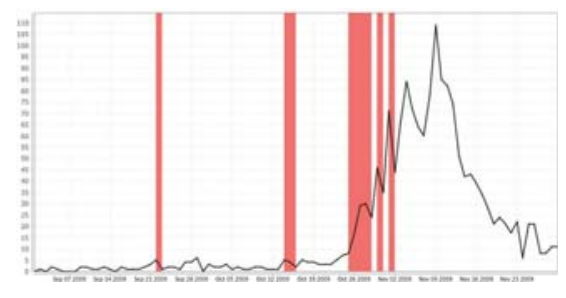

2009 H1H1 outbreak with alerts generated by RecentMax

\section{Keywords}

aberration detection; algorithm; outbreak detection; surveillance; situational awareness

\section{*Dave Carter}

E-mail: david.carter@cnrc-nrc.gc.ca 